\title{
Research on Charge and Discharge Control System of Hybrid Bicycle
}

\author{
Liting Geng ${ }^{1, a^{*}}$, Bo Chen ${ }^{1, b}$ and Xiangfan Piao ${ }^{1, c}$ \\ ${ }^{1}$ YanBan University YanJi JiLin Province, China \\ adieer918@163.com, bcb_ybu@163.com, cpxf@ybu.edu.cn
}

Keywords: Hybrid power bicycle; Energy recycle; Control system; Hub motor

\begin{abstract}
For the further development of hybrid technology and energy recovery technology application to improve energy efficiency. Research on hybrid bicycle control system comprising a brushless DC hub motor control and energy recovery process, the system selects STC12C5620AD as the control for brushless DC hub motor design motor control circuit, and proposed a system applicable to the energy recovery strategy, two-way flow of energy in the battery to the motor. Experimental results show that the system control circuit efficiency up to $80 \%$ for this overall braking energy recovery test of up to $15 \%$, indicating the effectiveness of the energy recovery strategy. Comprehensive tests show that the system is easy to use, reliable, good stability, can be used as energy-saving vehicles.
\end{abstract}

\section{Introduction}

With the improvement on people's living standards, motor vehicles seem to be essential for each household a means of transport ${ }^{[1]}$. At the same time, most areas and cities in China frequent severe mist haze weather, air monitoring to report shows that particulate pollutants seriously exceeded, with the use of motor vehicles increased year by year, vehicle emissions have become the main source of air pollution one ${ }^{[2,3]}$. And air quality to a certain extent, a direct impact on people's health and quality of life ${ }^{[4]}$. Therefore, to reduce the use of fuel consumption vehicles, instead of using envi-ronmentally friendly electric means of transport on the improvement of air quality and people's health protection has a great role in promoting ${ }^{[5,6]}$.

With the continuous development of automotive technology, environmental protection and energy conservation products more and more popular, and by the vast number of consumers love ${ }^{[7]}$. In recent years, hybrid technology and energy recovery technology has become an important re-search direction, at the same time, the development of new energy has also been the world's at-tention and favor ${ }^{[8]}$. The system through the collection and conditioning motors output signal, put forward an energy recovery strategy to achieve energy recovery or re-use ${ }^{[9]}$. It is very important to study the charge and discharge controls system suitable for hybrid bikes, which is very important to saving energy, reducing environmental pollution and alleviating urban traffic pressure ${ }^{[10]}$.

\section{System Composition and Structure}

The system mainly uses the STC12C5620AD single-chip microcomputer as the control core, the system through the input control signal respectively to realize the charge and the dis-charge two circulation circuit, realize the energy two-way flow. MCU to connect with the various functions of the interface parameters of the collection, and display the basic data.

As shown in Figure 1, the system mainly includes a discharge circuit, a charging circuit, a signal acquisition and conditioning circuit and a display part of four modules, wherein the discharge circuit includes an inverter and a driving circuit, the motor is controlled by the conduction signal of the switch tube, The circuit is generated by the motor after the rotation of the power, through the recti-fier boost circuit connected to the battery. 


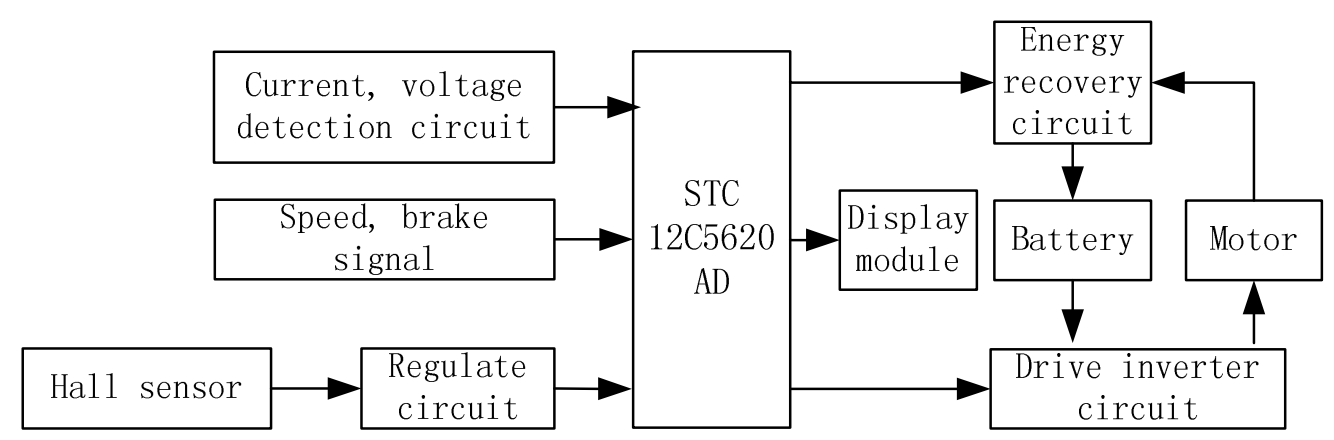

Figure 1. system hardware block diagram

Discharge circuit: mainly by the battery, drive inverter circuit and motor components. Accord-ing to the motor within the three-way signal to determine the current location of the motor, accord-ing to the output of six PWM control signal to control the conduction and cut off the switch, which indirectly control the motor phase current off, to achieve the motor commutation process, And completes the discharge process from the battery to the motor. Energy recovery circuit: mainly re-fers to the motor in the power generation mode, the system for the collection of motor output ener-gy, the motor output signals rectifier filter, and then through the boost circuit, through the PWM control battery charging current, to the motor to the battery Charging process. Signal acquisition and conditioning circuit: the acquisition signal mainly includes bus voltage, loop current, speed pulse, three-position signal and turn the handle and brake input signal. Which brakes and turn the main control signal is input to the single-chip, the collected current and voltage signals are mainly used as a basis for the normal operation of the protection system. Display module: The module is mainly on the current speed and the current battery remaining power display.

\section{System Hardware and Software Design}

Brushless DC Motor Control Circuit. The system discharge process is mainly by the DC power supply, change the inverter switch tube conduction sequence, so that the armature winding phase of the conduction sequence changes to achieve the normal operation of the motor. Whenever the rotor turns through the $60^{\circ}$ electrical angle, the controller changes the conduction sequence of the switch according to the po-sition of the rotor, thus changing the energization mode of the armature winding and the generated electromagnetic field, so that the motor keeps running continuously. Figure 2 for the brushless DC motors control circuit.

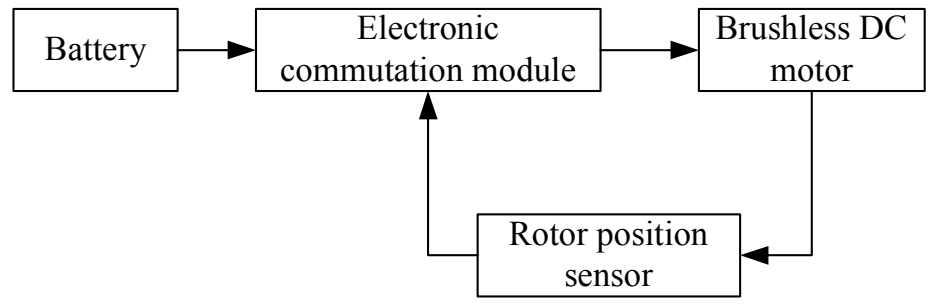

Figure 2. brushless DC motor control circuit

The electronic commutation module is mainly composed of the inverter circuit, which is the core of the motor control. Its physical circuit is composed of six switching tubes and special driving chip. The three-phase bridge powers inverter circuit is composed. The Hall element rotor position sensor is used to complete the phase of the brushless DC motor, which is divided into three Hall elements, distributed evenly among the circumference of the motor cover, so the Hall elements are different from each other by $120^{\circ}$ electrical angle.

Energy Recovery Circuit. The energy recovery process of the system is to include the bike in the normal driving, downhill or braking, according to the motor characteristics, the motor can act as a generator role, the use of motor generated back electromotive force as the energy recovery input, the energy recovery process additional Or the originally unwanted kinetic energy becomes the 
process of storing energy into the energy storage device.

The energy recovery circuit designed in this paper is mainly to store the output energy of the brushless DC motor into the battery. In this process need to rectify the output signal boost, through the PWM control charge current or directly through the energy consumption of energy consump-tion. Design resistance energy consumption This function is to the battery remaining capacity is relatively saturated, not only can reduce the use of mechanical brakes can protect the battery is not overcharge. Figure 3 is a block diagram of the energy recovery process.

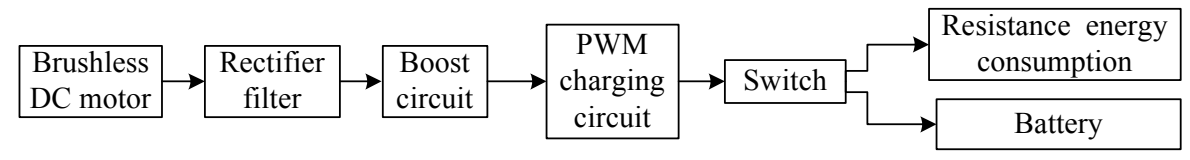

Figure 3. block diagram of energy recovery circuit

Single-Chip Interface Circuit. The system uses STC12C5620AD single-chip as the control chip, the system mainly by the chip control to achieve charging, discharge, detection, display and other functions. Where in the charging and discharging circuits are switched back and forth by switching. The single-chip circuit shown in Figure 4.

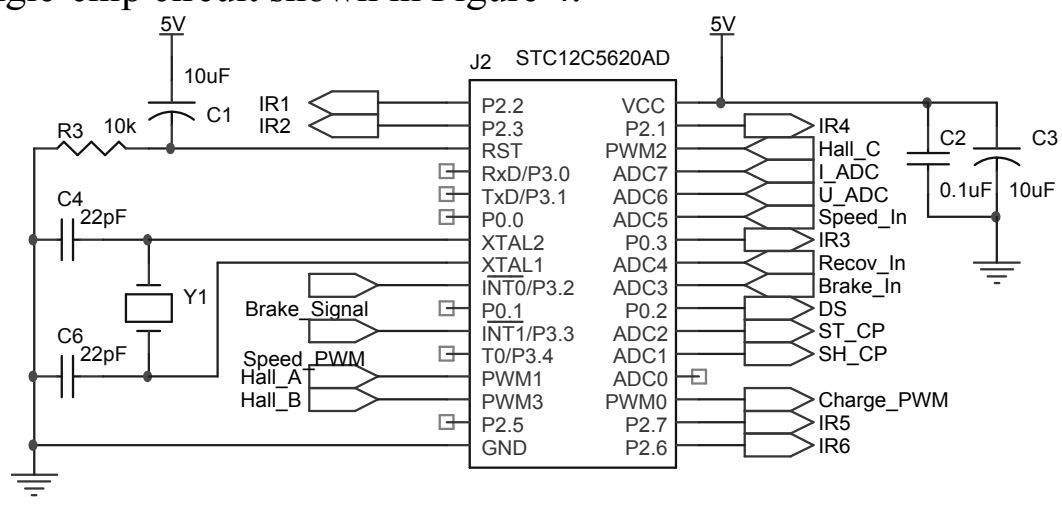

Figure 4. single-chip interface circuit

Figure 4, INT0 port input brake signal; ADC3 input brake strength signal; P0.2 port, P1.1 port and P1.2 port for the display module data input and control signals; PWM0 output charge control signal; PCA1 port, PCA2 port and PCA3 port are input to the motor three position signals; ADC6 port and ADC7 port input voltage and current detection signals; P2.2, P2.3, P0.3, P2.1, P2.7 and P2.6 Respectively, the output motor inverter circuit drive signal; ADC4 input energy recovery strength; ADC5 input speed signal; INT1 input measured speed pulse signal.

Charge Control Strategy. This paper is mainly for Ping Road, downhill, brake three different work conditions designed energy recovery program. The parameters in the energy recovery scheme are based on their own characteristics. Since the system controls the size of the charge current by means of the PWM, the current is determined by changing the duty cycle Di of the PWM or by directly giving a charge current Ii constant in the energy recovery scheme.

Flat road when using conventional energy recovery. PWM duty cycle constant, current fluctuations. Given a certain charge duty cycled, the specified duty cycle is specified

$$
D_{i}=\beta D_{0}
$$

Where D0 is the initial duty cycle, set to 0.1 , and $\beta$ is an adjustable coefficient between 1 and 1.5 , the default state is 1 , which can be set according to the user's personal preference.

Downhill using dynamic energy recovery. PWM duty cycles showed a linear change, current fluc-tuations. Since the acceleration a generated by the gravitational component will cause the system speed to increase linearly, the motor power generation will increase linearly. Allow the charge duty cycle according to the acceleration along the linear changes, and the specified duty cycle: 
$D_{i}=\beta D_{0}(1+\lambda a T)^{i}$

Where $\mathrm{T}$ is the velocity sampling period, $\mathrm{i}$ is the number of sampling periods in an acceleration process, beyond which the $\mathrm{i}$ is cleared and then recalculated, and $\lambda$ is the acceleration time coeffi-cient. Take the usual slope of $20^{\circ}$ when the acceleration, $\lambda$ take 0.02 .

Brake with brake energy recovery. Deceleration a 2 constant, PWM fluctuation. In this case, it is necessary to realize the purpose of decelerating the bicycle. Therefore, when the energy recovery is taken as the reference value of the deceleration, the constraint condition for the braking energy recovery is

$$
\left\{\begin{array}{l}
a_{2}=k_{z} a_{0} \\
I=\min \left(I_{t h}, I_{i}\right)
\end{array}\right.
$$

Which a0 take $1 \mathrm{~m} / \mathrm{s} 2$, kz for the brake strength, after the conversion of the Single-chip after the range of 0 to 2, a 2 for the bicycle deceleration. Ii for the braking energy recovery can reach the charging current, Ith for the safe charge current, I is the final recovery strategy selected charging current.

Experiment. This part of the experiment is based on flat road, slope and brake three different work carried out, respectively, the conventional energy recovery, dynamic energy recovery and braking energy recovery experiments. During the experiment, the notebook computer is connected with the meas-uring end of the hybrid bicycle through the data acquisition card. The experimental data is displayed and stored in the Labview directly through the data acquisition card. Figure 5 for the experimental process of data acquisition processes diagram.

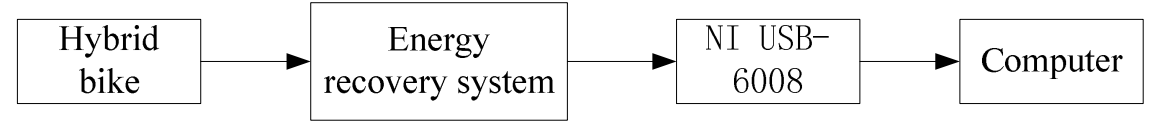

Figure 5. experimental data acquisition flow chart

By observing the lithium battery of a variety of works under the charge current size to reflect the effectiveness of energy recovery strategy. During the experiment, the speed of the hybrid bicy-cle is about $20 \mathrm{~km} / \mathrm{h}$, its weight is $15.5 \mathrm{KG}$, and the weight is about $65 \mathrm{KG}$. Figure 6 for the flat road energy recovery effect, the abscissa represents the Labview measurement program running rela-tive time. Can be seen from the figure, the charging current is relatively stable, in the course of travel did not appear any abnormal phenomenon. And, with the speed of the upgrade, you can see that the charging current also has a rising trend.

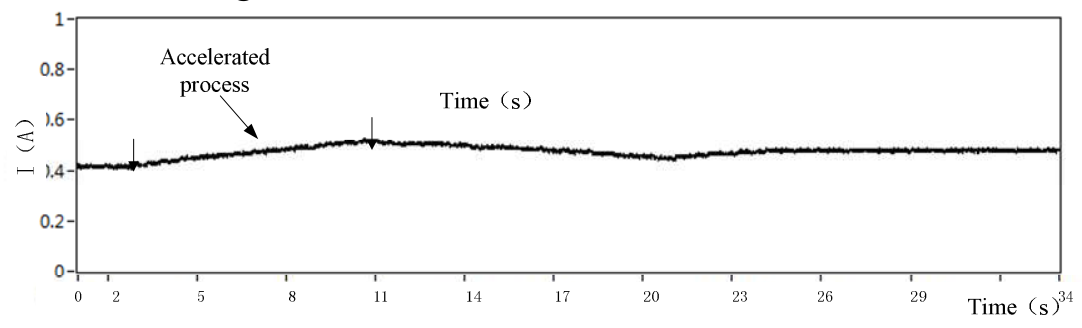

Figure 6 . battery charge current waveform (flat road)

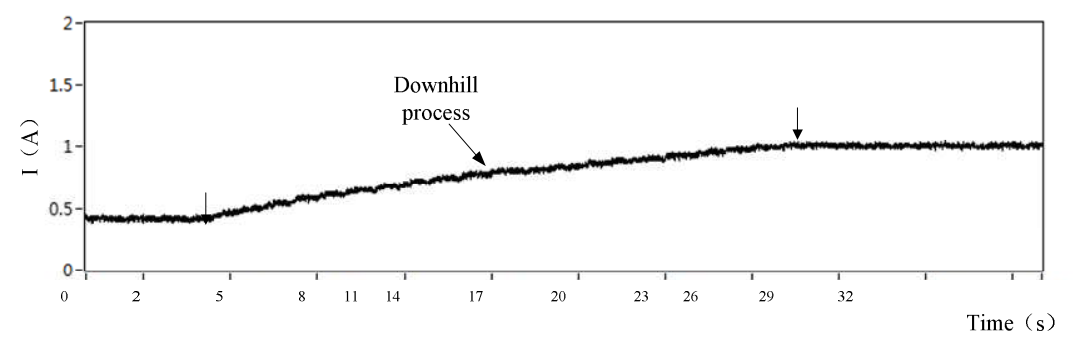

Figure 7. Battery charge current waveform (downhill) 
Downhill charging experiment selected in the slope of about $20^{\circ}$ of the road, because the bike in the downhill section of the operation, so the charge duty cycle continues to increase, so the charg-ing current also increases with the speed. It can be seen from Fig. 7 that the longer the acceleration time and the higher the charging current in the downhill process, because in the energy recovery strategy, the continuous acceleration process will cause the charge duty cycle to continue to increase, indicating the recovery strategy Effectiveness.

Figure 8 for the brake energy recovery effect map, can be seen from the figure, the experimental braking time is about $5 \mathrm{~s}$, the average charge current of $1.8 \mathrm{~A}$, in the braking energy recovery, the system charge current changes can be divided into A, B, C three processes, process $\mathrm{A}$ brake from weak to strong; process $\mathrm{B}$ as the main brake, to achieve deceleration; process $\mathrm{C}$ is the end of the brake.

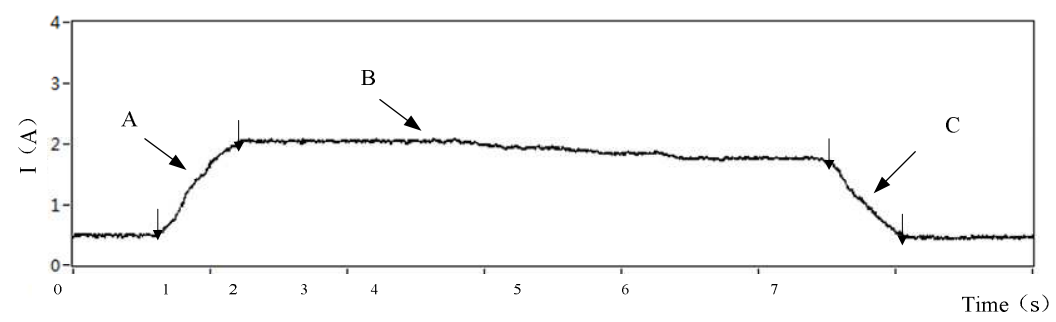

Figure 8 . Battery charge current waveform (brake)

So far, there is no uniform calculation standard and definition of the recovery of energy. In the pro-cess of energy recovery of both flat and downhill, there are external forces other than electric pow-er, so the recovery rate of the braking energy recovery process is analyzed. However, each of the researchers for different research objects will be based on the actual situation of the actual treat-ment.

The evaluation method of this system is mainly based on the energy recovery efficiency $\eta$ reg as the system evaluation index, calculated by the formula (4-1) by calculating the relationship between the kinetic energy loss of the system and the energy obtained by the battery.

$$
\eta_{\text {reg }}=\frac{E_{\text {reg }}}{E_{\text {recoverable }}} \times 100 \%
$$

Among them, Erecoverable is the energy recovery in the whole process, that is, the energy lost by the system. Erecoverable's formula can be expressed by the amount of kinetic energy,

$$
E_{\text {recoverable }}=\Delta E=\frac{1}{2} m v_{0}^{2}-\frac{1}{2} m v_{1}^{2}
$$

Where $\mathrm{v} 0$ and $\mathrm{v} 1$ denote the initial speed of the brake and the speed at the end of the braking, re-spectively. Ereg in equation (4-1) is the recovered energy, and its expression is:

$$
E_{\text {reg }}=\frac{\sum_{t_{0}}^{t_{1}} u(t) i(t)}{f}
$$

Where $\mathrm{f}$ is the sampling period, $\mathrm{u}(\mathrm{t})$ and $\mathrm{i}(\mathrm{t})$ are the voltage and current values corresponding to the sampling time in the braking process, respectively. T0 is the sampling time at which the calcu-lation starts, and $t 1$ calculates the end of the sampling time. Its specific value is through the Labview sampling data display chart directly derived and calculated. Efficiency $\eta$ represents the efficiency of the energy recovery circuit, the expression is: 


$$
\eta=\frac{P_{\text {reg }}}{P_{\text {motor }}}=\frac{\frac{E_{r e g}}{\Delta t}}{\sum_{t_{0}}^{t_{1}} u_{2}(t) i_{2}(t) \frac{T}{\Delta t}}=\frac{E_{\text {reg }}}{\sum_{t_{0}}^{t_{1}} u_{2}(t) i_{2}(t) T} \times 100 \%
$$

Where $\mathrm{T}$ is the sampling period, i2 $(\mathrm{t})$ is the motor armature current, $\mathrm{u} 2(\mathrm{t})$ is the DC voltage after the motor is rectified.

After several experiments, several sets of data were selected for analysis. The experimental data and the results are shown in Table 1. The experimental process is to simply consider the brake recovery performance, select the flat road. The selected six sets of experimental data can be di-vided into three groups according to the different start speed and the end speed.

Table 1 Braking energy back to data analysis

\begin{tabular}{cccccccc}
\hline $\begin{array}{c}v_{0} \\
(\mathrm{~m} / \mathrm{s})\end{array}$ & $\begin{array}{c}v_{1} \\
(\mathrm{~m} / \mathrm{s})\end{array}$ & $\begin{array}{c}\Delta t \\
(\mathrm{~s})\end{array}$ & $\begin{array}{c}\Delta E \\
(\mathrm{~J})\end{array}$ & $\begin{array}{c}P_{\text {motor }} \\
(\mathrm{w})\end{array}$ & $\begin{array}{c}E_{\text {reg }} \\
(\mathrm{J})\end{array}$ & $\eta_{2}$ & $\eta_{\text {reg }}$ \\
\hline 5.8 & 2.2 & 3.8 & 1159.2 & 53.8 & 181.3 & $88.7 \%$ & $15.6 \%$ \\
5.8 & 2.2 & 4.0 & 1159.2 & 54.2 & 189.6 & $87.5 \%$ & $16.4 \%$ \\
6.4 & 2.5 & 4.8 & 1397.1 & 55.3 & 226.4 & $85.3 \%$ & $16.2 \%$ \\
6.4 & 2.5 & 5.2 & 1397.1 & 55.9 & 241.8 & $83.2 \%$ & $17.3 \%$ \\
7.3 & 2.6 & 6.4 & 1872.8 & 57.8 & 312.0 & $84.3 \%$ & $16.7 \%$ \\
7.3 & 2.6 & 6.8 & 1872.8 & 58.4 & 329.3 & $82.9 \%$ & $17.6 \%$ \\
\hline
\end{tabular}

It can be seen from the experimental data that the conversion efficiency $\eta 2$ from the motor to the battery is mainly determined by the efficiency of the boost circuit and the PWM charging circuit. The experimental data show that the energy conversion efficiency of the process can reach more than $80 \%$, and the braking energy the recovery rate can be basically stable at $15 \%$ or more.

\section{Conclusion}

In order to improve the energy utilization rate, this paper presents a charging and discharging con-trol scheme for brushless DC wheel motor based on hybrid bicycles. The system through the cor-responding hardware circuit to detect and drive the motor signal, through the energy recovery cir-cuit to achieve and control the battery charge, you can not affect the normal work of the system to achieve the energy in the motor and the battery between of the two-way flows process. This paper also presents an energy recovery strategy for hybrid systems. The strategy can be used to control the charging current of the battery and make timely adjustment when the motor is in the power generation state according to the different energy recovery scheme of the system according to the different working conditions of the system.

\section{References}

[1] M. Yamato. Eco-Vehicle Assessment System (Eco-VAS): a Comprehensive Environmental Impact Assessment System for the Entire Development Process. Toyota Technical Review. 2005, 54(1): 80 85.

[2] B. Randal. High-Voltage Battery Simulator and Test Systems Critical for Electric and Hybrid Vehicle Development: Essential for Conducting R and D, Performance and Durability Testing. Advanced Battery Technology. 2013, 49(12): 18 21.

[3] Q. Ning, D. Xuan, Y. Kim. Modeling and control strategy development for fuel cell hybrid vehicles. International Journal of Automotive Technology. 2010, 11(2): 229 238.

[4] D. Chao, S. Peng - Fei, Zhang Sen. Development of Novel Bipolar Nickel/Metal Hydride Batteries for Hybrid Electric Vehicles. Chin. J. Chem. 2005, 23(2): 33 36. 
[5] Y. Gao, L. Chen, Ehsani M. Investigation of the Effectiveness of Regenerative Braking for EV and HEV. SAE Transactions. 2000, 108(6): 3184 3190.

[6] X. Wang, R. Liu. Research on stability and minimum orifice area of hydraulic servo position control system. Journal of Zhejiang University Science. 2001, 2(4): 421 425.

[7] S. Çetin, A. V. Akkaya. Simulation and hybrid fuzzy-PID control for positioning of a hydraulic system. Nonlinear Dynamics. 2010, 61(3): 465 476.

[8] G. Ma, S. Ning, Y. Hu, J. Gao. Dynamic balance analysis of contamination control for hydraulic systems based on dynamic filtration ratio. Industrial Lubrication and Tribology. 2015, 68(1): 45 51.

[9] K. Seong Han, C. Nam. Hardware-in-the-loop simulations of an electric hydraulic power steering system for developing the motor speed map of heavy commercial vehicle. Proceedings of the Institution of Mechanical Engineers. 2015, 229(13): 1717 1731.

[10]A. Soimu, F. Stinga, D. Popescu. A comparative study between MP and DLQ control strategies for a flexible link. Acta Montanistica Slovaca. 2010, 15(1): 38. 